\title{
Effectiveness of Booster Measles-Mumps-Rubella Vaccination in Lower COVID-19 Infection Rates: A Retrospective Cohort Study in Turkish Adults
}

\author{
Erhan Yengil (D) \\ Yusuf Onlen (D) ${ }^{2}$ \\ Cahit Ozer (1D) \\ Mustafa Hambolat $\mathbb{D}^{3}$ \\ Mehmet Ozdogan (1D) \\ 'Department of Family Medicine, Hatay \\ Mustafa Kemal University School of \\ Medicine, Hatay, Turkey; ${ }^{2}$ Department of \\ Infectious Disease and Clinical \\ Microbiology, Hatay Mustafa Kemal \\ University School of Medicine, Hatay, \\ Turkey; ${ }^{3}$ Province Health Manager of \\ Hatay, Turkey; ${ }^{4}$ Head of Provincial Public \\ Health Department, Hatay, Turkey
}

Objective: The aim of this study was to investigate the effectiveness of booster vaccination of adults with measles-mumps-rubella in the COVID-19 infection rates.

Methods: In order to investigate this hypothesis, we tested COVID-19 positivity rate through PCR assay on the participants ( $n=245$; male), who had to share the same student accommodation together with the same dining hall to provide governmental service. Participants were divided into two groups based on their booster vaccination status with measles-mumps-rubella: the non-vaccinated group $(n=207)$ and the vaccinated group $(n=38)$. The rate of COVID-19 seropositivity, age, body mass index (BMI), active smoking and presence of comorbidity were also measured and recorded.

Results: All of the participants were healthy, and age distribution, comorbidity rates, active smoking status and BMI did not vary significantly among the two groups $(p=0.305, p=0.594$, $p=0.280$, and $p=0.922$, respectively). About $36.7 \%(\mathrm{n}=90)$ of the participants were found to be COVID-19 positive by PCR among which the non-vaccinated cases had higher rates of COVID-19 seropositivity than the vaccinated cases $(40.6 \%$ vs $15.8 \%)(\mathrm{OR}=3.6,95 \% \mathrm{CI}$ : $1.5-9.0, p=0.004)$.

Conclusion: Based on these results, we cautiously predict that immunity produced by MMR vaccination boosters may provide some degree of protection against COVID-19 in the adult population.

Keywords: COVID-19, live attenuated vaccines, booster vaccination, MMR

\section{Introduction}

Coronavirus disease 2019 (COVID-19), due to the new coronavirus (SARS-CoV-2) is an emerging pandemic disease, affecting millions of people worldwide. ${ }^{1}$ SARSCoV-2 is an enveloped, positive-sense, single-stranded RNA virus classified within the genus beta-corona virus in the Coronaviridae family, which has been shown to have $79 \%$ and $50 \%$ sequence similarity with SARS-CoV and MERS-CoV, respectively. ${ }^{2}$ This novel virus has been reported to cause severe lower respiratory tract infection in humans which often leads to deadly respiratory insufficiency. ${ }^{3,4}$ According to the WHO report on July 7, 2020, the number of confirmed cases reached almost 11.5 million and more than half a million of them died all around the world so far. ${ }^{5}$

The present pandemic of COVID-19 is increasing and expanding rapidly at an alarming rate around the world. However, the infection rate has varied greatly among countries ${ }^{6}$ and it has been speculated that this difference could be related
Correspondence: Erhan Yengil Hatay Mustafa Kemal University, Faculty of Medicine, Department of Family Medicine, Hatay, Turkey

Email dryengil@yahoo.com 
with routine live vaccination policies carried out in each country. ${ }^{6,7}$ For instance, the findings of an epidemiological research conducted by Gold, indicated that the number of COVID-19 cases are lower in countries where live viral vaccines, such as measles, mumps, rubella (MMR), were applied routinely at every age group including the adult population. ${ }^{8}$

Currently, enormous efforts are being devoted to develop a vaccine against this virus to control the pandemic. There has also been growing interest in the repurposing of existing vaccines owing to the possible difficulties in the development of a new vaccine targeting SARS-CoV-2. ${ }^{9}$ Despite the existence of unproven hypotheses on the protectiveness of live attenuated vaccines as MMR on COVID-19 disease, there is no clinical study investigating the protectiveness of the booster dose of those kind of vaccines against COVID-19 on adults. ${ }^{10-12}$

Right after the first case of COVID-19 in Turkey, we had a chance to investigate whether the booster vaccination (including MMR and varicella) may provide a meaningful protective effect against COVID-19 using cohort study, for which we obtained data from wellorganized military officers with the aim of launching some operational activities.

\section{Methods}

\section{Study Subjects}

The study participants were taken from the cohort of the military officers $(n=257)$ who were recruited by the Hatay Governorate. Subjects shared the same accommodation (military base) over the period March to June 2020. In March 2020, 38 soldiers were given booster live vaccinations including a booster dose of live attenuated vaccines (including MMR and varicella) and killed vaccines (including HAV, HBV, MenACWY and diphtheria-tetanus vaccine) for potential cross-border operations. These participants were selected as the vaccine cohort and rest of the soldiers $(\mathrm{n}=219)$ were selected as the non-vaccine cohort (Figure 1).

On May 2, 2020 a subject tested positive for the COVID-19 and all subjects (257), who shared the same accommodation, were scanned for the COVID-19 using the real-time transcriptase polymerasechainreaction (RTPCR) on nasal and pharyngeal swab in accordance with published guidelines. ${ }^{13}$ All subjects, whose PCR results were negative, were quarantined and monitored for 14 days. After the follow-up, the subjects were re-tested for
COVID-19. This retrospective cohort study was approved by the Hatay Mustafa Kemal University, NonInterventional Clinical Research Ethics Committee (approval number: 08-32/2020) and by the Ministry of Health of Turkey.

\section{Data Source}

A total of 245 participants (38 from the vaccine cohort group and 207 from the non-vaccine cohort) agreed to respond to the self-administered questionnaire, and were therefore included in this study. Each person was informed about the study and assigned a unique identification number. We obtained data on vaccine status for each participant from the provincial health directorate. We also obtained information on demographic (age), clinical data, body mass index (BMI), comorbidities, smoking habits and vaccination status from interviewer-administrated questionnaires.

\section{Statistics}

The data were analyzed using SPSS v. 21.0 software. (IBM Corporation, Armonk, NY, USA). Continuous variables were tested for normality with onesample Kolmogorov-Smirnov test. Nominal variables were compared using chi-squared test and Fisher's exact test between the groups, while Mann-Whitney $U$-test was used for continuous variables. For all statistical data, $p<0.05$ was considered significant. Figure 2 was plotted using GraphPad Prism v 7.0d for Mac (GrapPad Software, La Jolla, CA, USA).

\section{Results}

The median age was 26 (23-53) years for the vaccinated group and 27 (21-54) years for the non-vaccinated group $(p=0.305)$.

The BMI of the vaccinated group and non-vaccinated group was 24.82 (20.20-28.41) and 25 (19.05-30.58), respectively $(p=0.922)$. There was no past or present history of chronic disease in the vaccinated group, whereas six subjects $(2.9 \%)$ within the non-vaccinated group had chronic disease $(p=0.594)$. Regarding the smoking habits, $50 \%$ of the vaccinated subjects $(n=19)$ and $59.4 \%(n=123)$ of non-vaccinated subjects were active smokers $(p=0.280)$ (Table 1).

During the study period, a total of 90 participants (36.7\%) were diagnosed with COVID-19 by RT-PCR. According to the PCR results, the significant difference for COVID-19 seropositivity was observed among vaccinated group $(n=6$; 


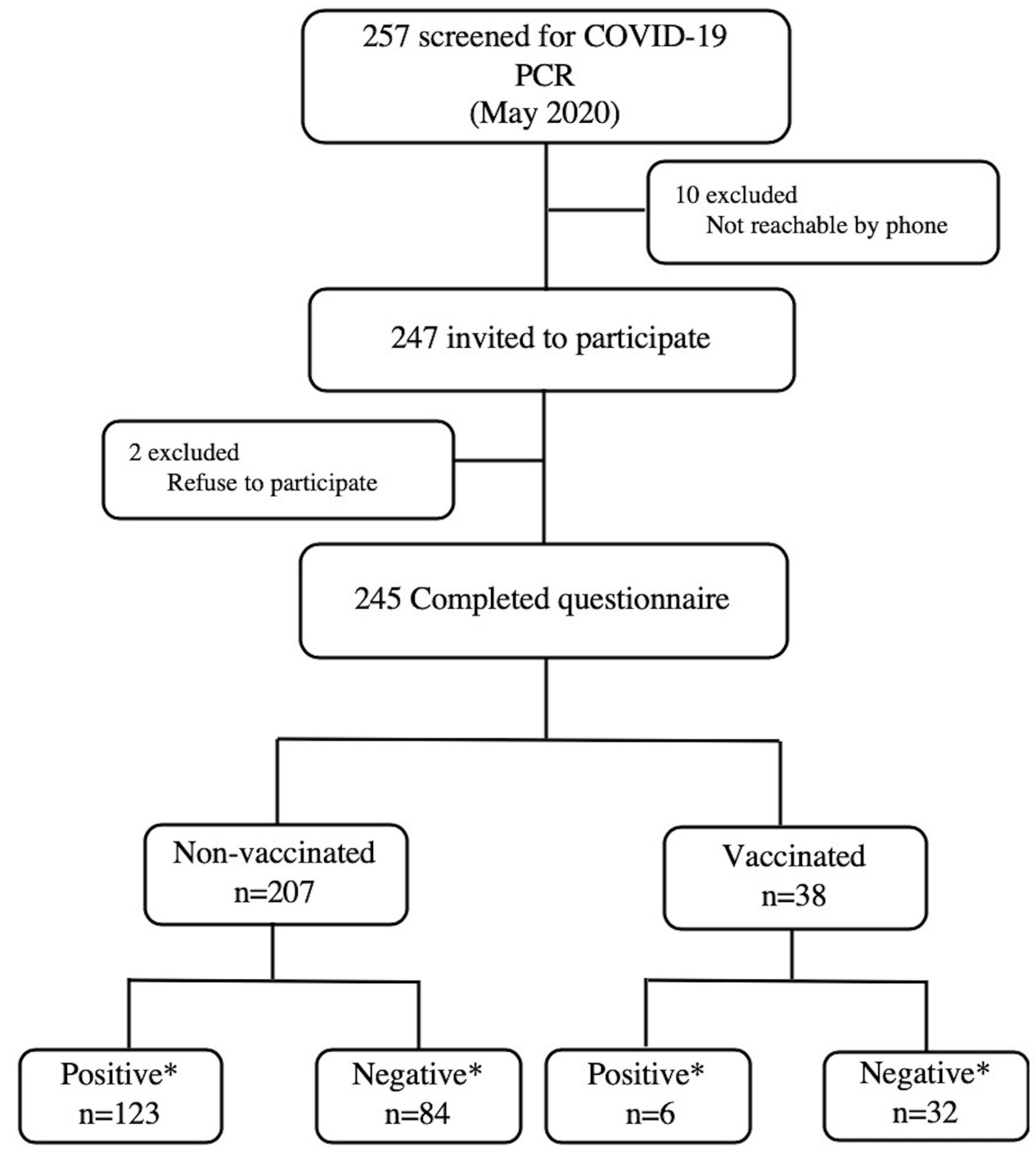

Figure I Flow chart of this study.

$15.8 \%)$ and non-vaccinated group $(\mathrm{n}=84 ; 40.6 \%)(\mathrm{OR}=3.6$, 95\%CI: $1.5-9.0, p=0.004$, Figure 2).

All COVID-19 positive patients had mild to moderate symptoms for which headache and fatigue (58.4-57.8\%, respectively) were most frequently identified (Figure 3 ). Although there were no severe cases, 11 subjects (10 from the non-vaccinated group; one from the vaccinated group) had shortness of breath; but it was mild and nonprogressive. Fever was reported more frequently in the non-vaccinated group compared to vaccinated group (29.7\% vs $2.6 \%$, respectively $p<0.05)$.

\section{Discussion}

Epidemiologically, children and the elderly are two groups with a high risk of viral respiratory infections. ${ }^{14}$ However, information from COVID-19 patients shows that children are less susceptible to the disease compared to adults and the elderly. ${ }^{15,16}$ This difference is explained by the trained immunity induced by frequent viral infections and routine live attenuated vaccines in children which led to a protective effect, not only on the targeted pathogen, but also on other unrelated pathogens for at least three months. ${ }^{10,11,16-18}$ However, it is not yet clear whether a live attenuated vaccine booster has a protective effect against the development of COVID-19 disease in adults. The results of the current study showed that nonvaccinated participants have a 3.6 times higher probability of developing COVID-19 disease than participants who recently (within the last three months) received a booster dose of live viral vaccines (including MMR and varicella).

Generally, vaccines provide elicit a specific immune response against targeted pathogen. However, live 


\section{COVID-19 RT-PCR results}

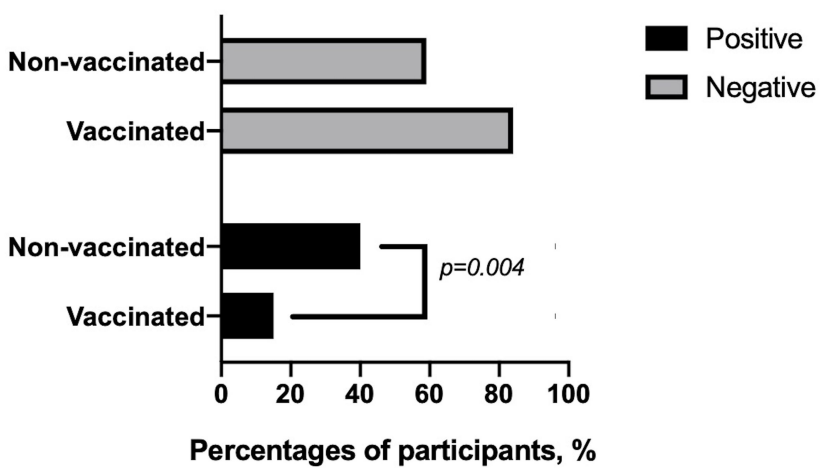

Figure 2 The comparison of COVID-19 RT-PCR results between the groups. Abbreviation: RT-PC, reverse transcriptase polymerase chain reaction.

attenuated vaccines (MMR, rotavirus, smallpox, varicella, BCG) were capable of inducing trained immunity, which mediates protection to unrelated pathogens, too. ${ }^{19}$ Consequently, it is thought that at least one of those two live viral vaccines (varicella, MMR) might have had a protective effect against the development of COVID-19 disease in our study.

While the SARS-CoV-2 virus is an RNA virus, varicella vaccine consists of a human DNA virus (varicella-zoster virus). In contrast, based on the current literature there has been no evidence as to whether the varicella vaccine could provide protection against COVID-19. ${ }^{20}$ However, the MMR vaccine consists of weakened enveloped RNA viruses. The spike (S) glycoprotein of SARS-CoV-2 virus plays a key role in the viral entry into the cells, which makes it an important target for developing new vaccines. ${ }^{9}$ Due to the close similarities between the spike glycoprotein sequences of SARS-CoV-2 and viruses in MMR vaccine (the measles, mumps, and rubella spike glycoprotein sequences with a rate of $32 \%, 31 \%$ and $33 \%$, respectively), MMR vaccine-induced antibodies might provide cross protection against COVID-19. ${ }^{11,21}$ Furthermore considering that the measles virus has similar cell entry and replication mechanisms, similar transmission level and clinical findings, in addition to the structural similarity with the SARS-CoV-2 virus containing in the MMR vaccine, this live vaccine appears to be more likely to be MMR. ${ }^{21}$

A previous experimental study by Escriou et al provide concrete evidence that receiving a booster MMR vaccine may offer protection against SARS-CoV infection. In the same study the researchers evaluated the effectiveness of a viral vector vaccine containing the attenuated measles virus against $\mathrm{SARS}-\mathrm{CoV}$ infection. In this study it has been presented that the neutralizing antibody titers formed after two successfully applied immunizations have reached a level of complete protection against experimental intranasal SARS-CoV infection on rats. In this regard, they suggested that the recombinant measles virus vaccine can be used for the immunization of both adults and children in new SARS-CoV outbreaks that may occur in future. ${ }^{22}$ Even though the presence of a great similarity between SARS-CoV-2 and SARS-CoV was proven before, there is no immunologic study showing whether the same effect on SARS-CoV-2 could be provided with MMR vaccine or not.

The possible role of killed vaccines (including hepatitis B, hepatitis A, Men ACWY and tetanus-diphtheria (Td) vaccines) administered at the same time as live vaccines (including MMR and varicella vaccines) in mediating this possible protection from COVID-19 disease cannot be ruled out in the current study. However, the split inactivated vaccines tend to be less immunogenic compared to whole viral particles in live vaccines. ${ }^{23}$ This makes it harder to claim that these killed vaccines may contribute to partial protection, but still possible.

Epidemiological data from several studies suggest a correlation between receiving a booster dose of MMR vaccine may be associated with a lower severity of COVID-19 disease. ${ }^{8}$ But the present study failed to show this association. All COVID-19 positive patients had mild to moderate disease. This inconsistency may be due to the fact that participants from both groups were in the same

Table I The Comparison of Demographic Variables Between the Groups

\begin{tabular}{|l|c|c|c|}
\hline & Vaccinated Group (n=38) & Non-Vaccinated Group (n=207) & p-value \\
\hline Age $($ range) & $26(23-53)$ & $27(21-54)$ & 0.305 \\
BMI $\left(\mathrm{kg} / \mathrm{m}^{2}\right)$ & $24.82(20.2-28.4 \mathrm{I})$ & $25(19.05-30.58)$ & 0.922 \\
Comorbidity & $0 / 38$ & $6 / 201$ & 0.594 \\
Active smooking & $19 / 19$ & $123 / 84$ & 0.280 \\
\hline
\end{tabular}

Note: Data are expressed as median (min-max), or $n(y e s) / n(n o)$.

Abbreviations: BMI, body mass index; PCR, polymerase chain reaction. 


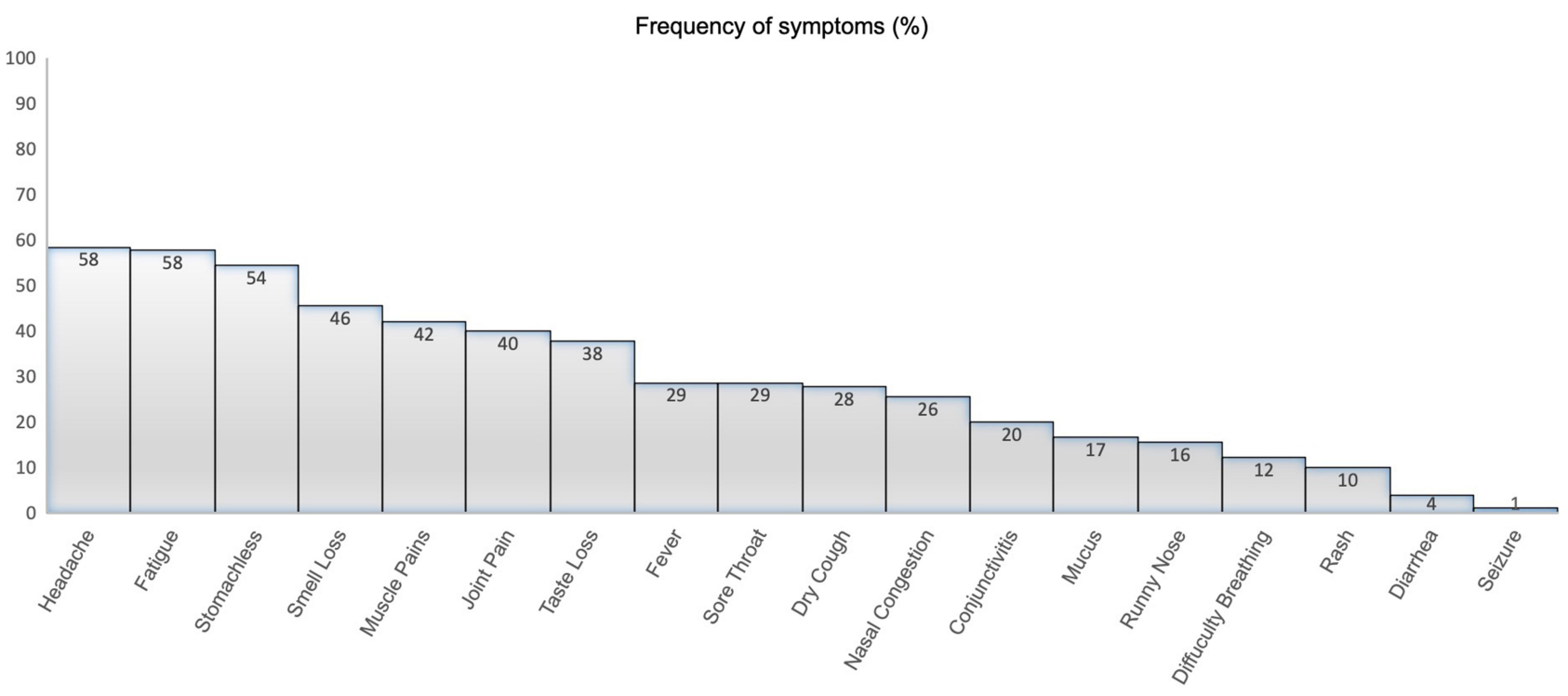

Figure 3 Frequency of symptoms reported in COVID-19 positive cases.

race, gender, with a similar comorbidity rate and both groups consisted of younger adults. Another possible explanation for this is the small number of vaccinated COVID-19 positive cases, which limits our ability to draw conclusions about how vaccination affects the clinical course.

Due to the possible difficulties in the development of a new vaccine targeting SARS-COV-2, it is thought that clinical studies on live attenuated vaccines will continue or even gain momentum in the near future. ${ }^{9,24}$

\section{Limitations}

It is certain that this research has some limitations. One of the most important limitations is that, all subjects are young adults. Therefore, this result cannot be generalized to other age groups. Another important limitation in this study is that it is not clear exactly which vaccine may have been effective in this partial protection. Prospective randomized controlled trials are needed to understand which vaccine is the main protective one. Also the antibody status not only to SARSCoV-2 (which is not mentioned at all), but also to the pathogens in the formula of vaccines that should be tested.

\section{Conclusion}

Based on these results, we cautiously predict that immunity produced by MMR booster vaccination may make adults less susceptible to COVID-19.

\section{Declaration}

Ethical statement: Local Research Ethics Committee $(2020 / 08 ; 32)$ in accordance with the Declaration of Helsinki.

\section{Acknowledgment}

The authors would like to thank HMKU for providing English editing service.

\section{Disclosure}

The authors report no conflicts of interest in this work.

\section{References}

1. Jin Y, Yang H, Ji W, et al. Virology, epidemiology, pathogenesis, and control of COVID-19. Viruses. 2020;12(4). doi:10.3390/v12040372.

2. Lu R, Zhao X, Li J, et al. Genomic characterisation and epidemiology of 2019 novel coronavirus: implications for virus origins and receptor binding. Lancet. 2020;395(10224):565-574. doi:10.1016/S01406736(20)30251-8

3. Perlman S. Another decade, another coronavirus. $N$ Engl $J$ Med. 2020;382(8):760-762. doi:10.1056/NEJMe2001126

4. Cascella M, Rajnik M, Cuomo A, Dulebohn SC, Di Napoli R. Features, Evaluation, and Treatment of Coronavirus. Treasure Island (FL): StatPearls; 2020.

5. World Health Organization. Novel Coronavirus (2019-nCoV) Situation Report-170; 2020.

6. Team CC-R. Geographic differences in COVID-19 cases, deaths, and incidence - United States, February 12-April 7, 2020. MMWR Morb Mortal Wkly Rep. 2020;69(15):465-471. doi:10.15585/mmwr. mm6915e4

7. Anbarasu A, Ramaiah S, Livingstone P. Vaccine repurposing approach for preventing COVID 19: can MMR vaccines reduce morbidity and mortality? Human Vaccines immunotherapeutics. 2020;16 (9):2217-2218. 
8. Gold JE. MMR vaccine appears to confer strong protection from COVID-19: few deaths from SARS-CoV-2 in highly vaccinated populations. 2020. doi:10.13140/RG.2.2.32128.25607

9. Caddy S. Developing a vaccine for covid-19. BMJ. 2020;369:m1790. doi:10.1136/bmj.m1790

10. Lyu J, Miao T, Dong J, Cao R, Li Y, Chen Q. Reflection on lower rates of COVID-19 in children: does childhood immunizations offer unexpected protection? Med Hypotheses. 2020;143:109842. doi:10.1016/j.mehy.2020.109842

11. Dhochak N, Singhal T, Kabra SK, Lodha R. Pathophysiology of COVID-19: why children fare better than adults?. Indian J Pediatr. 2020;87(7):537-546.

12. Sidiq KR, Sabir DK, Ali SM, Kodzius R. Does early childhood vaccination protect against COVID-19? Front Mol Biosci. 2020;7:120. doi:10.3389/fmolb.2020.00120

13. World Health Organization. Clinical management of COVID-19: interim guidance. (No. WHO/2019-nCoV/clinical/2020.5).

14. Rogier van Doorn HYH. Viral Respiratory Infections. Hunter's Trop Med Emerg Infect Dis. 2020;284-288.

15. Dong YMX, Hu Y. Epidemiology of COVID-19 among children in China. Pediatrics. 2020;145(6). doi:10.1542/peds.2020-0702.

16. Moorlag S, Arts RJW, van Crevel R, Netea MG. Non-specific effects of BCG vaccine on viral infections. Clin Microbiol Infect. 2019;25 (12):1473-1478. doi:10.1016/j.cmi.2019.04.020

17. Kleinnijenhuis J, Quintin J, Preijers F, et al. BCG-induced trained immunity in NK cells: role for non-specific protection to infection. Clin Immunol. 2014;155(2):213-219. doi:10.1016/j.clim.2014.10.005
18. Fidel PL Jr Noverr MC. Could an unrelated live attenuated vaccine serve as a preventive measure to dampen septic inflammation associated with COVID-19 infection? mBio. 2020;11(3). doi:10.1128/ mBio.00907-20

19. Covian C, Retamal-Diaz A, Bueno SM, Kalergis AM. Could BCG vaccination induce protective trained immunity for SARS-CoV-2? Front Immunol. 2020;11:970. doi:10.3389/fimmu.2020.00970

20. Blok BA, Arts RJ, van Crevel R, Benn CS, Netea MG. Trained innate immunity as underlying mechanism for the long-term, nonspecific effects of vaccines. J Leukoc Biol. 2015;98(3):347-356. doi:10.1189/ jlb.5RI0315-096R

21. Young A, Neumann B, Mendez RF, et al. Homologous protein domains in SARS-CoV-2 and measles, mumps and rubella viruses: preliminary evidence that MMR vaccine might provide protection against COVID-19. medRxiv. 2020. doi:10.1101/ 2020.10.28.20221747

22. Escriou N, Callendret B, Lorin V, et al. Protection from SARS coronavirus conferred by live measles vaccine expressing the spike glycoprotein. Virology. 2014;452-453:32-41. doi:10.1016/j. virol.2014.01.002

23. Ortbals DW, Liebhaber H. Comparison of immunogenicity of a whole virion and a subunit influenza vaccine in adults. $J$ Clin Microbiol. 1978;8(4):431-434.

24. Amanat F, Krammer F. SARS-CoV-2 Vaccines: status Report. Immunity. 2020;52(4):583-589. doi:10.1016/j.immuni.2020.03.007
International Journal of General Medicine

\section{Publish your work in this journal}

The International Journal of General Medicine is an international, peer-reviewed open-access journal that focuses on general and internal medicine, pathogenesis, epidemiology, diagnosis, monitoring and treatment protocols. The journal is characterized by the rapid reporting of reviews, original research and clinical studies

\section{Dovepress}

across all disease areas. The manuscript management system is completely online and includes a very quick and fair peer-review system, which is all easy to use. Visit http://www.dovepress.com/ testimonials.php to read real quotes from published authors. 